\title{
Um estudo de marketing aplicado ao setor madeireiro de Rio Branco, Estado do Acre, 1995
}

\author{
Z \\ $\mathrm{J}_{\text {OÃo }} \mathrm{C}_{\text {arlos }} \mathrm{G}_{\text {arzel }}$ Leodoro da $_{\text {ILVA }}{ }^{(2)}$
}

\section{RESUMO}

Este trabalho apresenta uma análise sobre a prática dos princípios de marketing adotados em 1995 pelo setor madeireiro em Rio Branco, no Estado do Acre. Esta pesquisa investigou 39 serrarias, 43 indústrias de móveis e 16 depósitos revendedores de madeira serrada localizados nesta cidade. Os principais aspectos considerados na análise foram: (1) ambiente de marketing; (2) composto de marketing; (3) mercados alvo e (4) estratégia de marketing. Algumas das conclusões obtidas foram: esforços de marketing, junto às firmas de construção civil locais, devem ser implementados para assegurar uma demanda para o produto madeireiro acreano; o setor madeireiro local necessita de medidas administrativas, técnicas-operacionais e políticas para o sucesso de um programa de marketing em suas firmas e a falta de uma prática do manejo florestal, pelas firmas madeireiras locais, pode ser um obstáculo de mercado às serrarias de Rio Branco.

Palavras-chave: Indústria madeireira, marketing de produtos madeireiros, economia florestal, Floresta Tropical Úmida, Estado do Acre.

\section{ABSTRACT}

A marketing study applied to the wooden sector of Rio Branco, State of Acre-Brazil, 1995. This paper presents an analysis on the practice of marketing principles adopted, in 1995, by the timber sector in Rio Branco (Capital of State of Acre, located in western Amazon-Brazil). The study covered 39 sawmills, 43 furniture manufacturers and 16 timber retailers located in this city. The main aspects considered in the analysis were: (1) marketing environment; (2) marketing mix; (3) target market and (4) marketing strategies.

\footnotetext{
${ }^{1}$ Engenheiro Florestal, M.Sc., pesquisador da Fundação de Tecnologia do Estado do Acre (FUNTAC) e estudante de Doutorado na Universidade Federal do Paraná (UFPR)

${ }^{2}$ Engenheiro Florestal, Dr., Professor Adjunto na Universidade Federal do Paraná (UFPR)
} 
Some of the conclusions obtained were: Practices of marketing, between local construction firms and sawmill must be established in order to develop a demand for Acrean wooden product; local timber sector needs some management, technical and policies actions in order to generate a good marketing program for their sawmills and the non use of forest management by local sawmills cam became a constraint for local timber firms as trade with consumers abroad of Acre.

Key words: Timber industry, marketing of forest product, forest economics, Tropical Moist Forest, Acre, Brazil

\section{INTRODUÇÃO}

O setor madeireiro de Rio Branco, capital do Estado do Acre, apresenta-se com características bem peculiares. Por um lado, este segmento produtivo tem um grande potencial quanto a oferta de matéria-prima madeireira (segundo AMARO, SILVA \& BRAZ, 1993, este Estado conta, ainda, com 93\% de sua superfície com cobertura florestal original). Por outro lado, este setor além tem sofrido com a diminuição significativa no número de suas indústrias.

\section{OBJETIVO}

Este artigo objetiva apresentar os resultados de uma pesquisa sobre marketing dos produtos madeireiros no Município de Rio Branco no Estado do Acre.

Os objetivos específicos compreenderam os seguintes tópicos:

a) Identificar o ambiente de marketing de produtos madeireiros em Rio Branco;

b) Analisar o uso do composto de marketing pelo setor madeireiro de Rio Branco;

c) Identificar os mercados-alvo do setor madeireiro de Rio Branco; e,

d) Subsidiar uma estratégia de marketing para produtos madeireiros em Rio Branco.

\section{REVISÃO DE LITERATURA}

\section{Conceitos de marketing aplicados ao setor florestal}

LINTU (1986 a, b) afirma que o marketing, como uma ciência, é relativamente jovem e a sua aplicação nas indústrias florestais é mais recente ainda. Porém, pode-se considerar que ele tem uma função básica crucial ligando todos os estágios na cadeia produtiva florestal. 
No atual processo de "globalização da economia" COHEN \& SMITH (1992) definem marketing internacional quando visa-se segmentos específicos de consumidores com linhas de produtos distintas e desenvolvidas para cada segmento e marketing global, por sua vez, quando não se considera aspectos geográficos, exportando-se uma única linha de produtos padronizados para todos os segmentos.

O conceito de estratégia de marketing teve sua origem no trabalho de ANSOFF $^{3}$, e foi posteriormente influenciado por SHIRLEY et al. ${ }^{4}$ e WEBSTER $^{5}$. Estes conceitos, aplicados na "interface" entre a firma e seu ambiente (consumidores, competidores e acionistas), tem sido muito discutido ultimamente e suas estratégias determinam-se considerando produtos, consumidores, mercado alvo e vantagem competitiva (NIEMELA \& SMITH, 1996).

Segundo SHIRLEY et al. ${ }^{6}$, citados por NIEMELA \& SMITH (1996), o marketing envolve questões fundamentais de gerenciamento considerando itens desde o "mix" de marketing até um conceito mais extensivo que visa obter vantagem competitiva.

PAUN, SHOOK \& SCHREUDER (1996) enfatizam que estratégias inovadoras de negócios são essenciais também para firmas florestais que visam melhorar sua competitividade e criar novos mercados. Esta situação deve-se às necessidades de ganho substancial nas vantagens operacionais relativas, existência de uma rivalidade global intensa, flutuações severas na oferta de matéria-prima e uma pressão crescente dos consumidores para valores maiores dos produtos.

Além disso, segundo JAWORSKI \& KOHLI ${ }^{7}$ e SLATER \& NAVER ${ }^{8}$, citados por PAUN, SHOOK \& SCHREUDER (1996), as empresas florestais, a nível mundial, abandonaram a filosofia gerencial orientada à produção (produzir primeiro e depois buscar compradores) e adotaram uma ação de trabalho voltada ao mercado (identificar primeiro o que os seus clientes necessitam e depois desenvolver processos para atender a essas necessidades).

Conhecendo o mercado, o administrador de uma indústria madeireira pode melhor conduzir o seu povoamento florestal. Este conhecimento, que de forma crescente vem sendo difundido nas escolas de engenharia florestal, permite que o administrador, com os produtos da floresta atenda a novos

\footnotetext{
${ }^{3}$ ANSOFF, H.I. Corporate strategy. New York: McGraw-Hill, 158 p. 1965.

${ }^{4}$ SHIRLEY, R.; PETER, M. \& EL-ANSARY, A. A. Strategy and policy formation - a multifuncional orientation. 2 ed. New York: Wiley, 286 p. 1981.

${ }^{5}$ WEBSTER JR, E. Industrial marketing strategy. New York: Wiley, 279 p. 1979.

${ }^{6}$ SHIRLEY, R.; PETER, M. \& EL-ANSARY, A. Ibid.

${ }^{7}$ JAWORSKI, B.J. \& KOHLI, A.K.. 1993. Market orientation: Antecedents and consequences. Journal of Marketing. 57(3):53-70.

${ }^{8}$ SLATER, S.F. \& NARVER, J.C. 1994. Does competitive environment moderate the marketing orientation-performance relationship? Journal of Marketing. 58(1): 46-55.
} 
mercados e a uma demanda por bens madeireiros específicos (PAUN, SHOOK \& SCHEUDER, 1996).

LAFLEUR, SINGHI \& MAcDIKEN (1996) salientam a crescente compreensão do valor do marketing para se administrar sistemas florestais e agroflorestais.

A falta do conhecimento básico sobre marketing é a maior ameaça para a silvicultura em grande escala na Índia, ressalta PATNAIK, (1996).

EASTIN (1996) lista avaliações de aumento no ganho com informações de mercado e pela certificação de operações florestais sustentáveis, desenvolvimento de mercado para espécies madeireiras menos conhecidas e desenvolvimento de mercado para produtos florestais não tradicionais como as estratégias de marketing adotadas pelos administradores florestais na África. Estas estratégias, orientadas nas preocupações dos consumidores e ambientalistas (quanto ao uso dos recursos florestais), podem ajudar a promover o uso sustentável das florestas tropicais.

Num passado recente, os objetivos e barreiras pouco influíram na formulação de estratégias de marketing internacional das empresas de papel e celulose no Brasil, onde as decisões ocorreram, principalmente, devido às oportunidades (SILVA, 1996).

\section{Estudos econômicos sobre o setor madeireiro de Rio Branco}

SAMANEZ MERCADO (1980), IBDF (1985), SANTOS (1986), MOUSASTICOSHVILY JR. (1991) e HUMMEL et al. (1994) estudaram a economia madeireira na Amazônia, porém não entraram em detalhe sobre o Estado do Acre.

AMARO, SILVA \& BRAZ (1993) analisaram o setor florestal do Estado do Acre, contudo não se aprofundaram sobre o seu mercado madeireiro.

IBDF (1984), DPRN (1986), FUNTAC (1990 e 1994), ARAUJO (1991 e 1993), FIEAC \& FUNTAC (1992), SEBRAE (1993), VIANA (1995) e OLIVEIRA (1997) abordaram tecnicamente a industrialização madeireira no Estado do Acre.

SILVA \& TOMASELLI (1997) enfocaram aspectos da comercialização de produtos madeireiros em Rio Branco. Porém, não analisaram os procedimentos de marketing adotados pelo setor madeireiro nesta região.

Os trabalhos citados mostram, claramente, que os aspectos de marketing não foram objeto de análise no Estado do Acre. Portanto, o presente artigo é fruto de uma pesquisa que foi desenvolvida para subsidiar estratégias de marketing para a comercialização de produtos madeireiros no Estado do Acre. 


\section{MATERIAL E MÉTODO}

Este artigo utiliza os dados usados por SILVA (1996) na sua pesquisa sobre o setor madeireiro de Rio Branco. A Tabela 1 sintetiza a amostragem adotada na coleta de dados.

Tabela 1 - Coleta de dados no setor madeireiro, Rio Branco/1995

Table 1 - Data collect of wood sector, Rio Branco/1995

\begin{tabular}{l|c|c|c|c}
\hline \multicolumn{1}{c|}{ Segmento } & Amostra piloto & Amostra definitiva & Pop. total & $\begin{array}{c}\text { Intensidade } \\
\text { amostral (\%) } \\
\text { Sample intensity } \\
(\%)\end{array}$ \\
\hline Serraria/Sawmill & 10 & Final sample & Total pop & $\begin{array}{c}\text { Sample } \\
\text { Marcenaria/Joinery }\end{array}$ \\
$\begin{array}{l}\text { Depósito/Timber } \\
\text { retail }\end{array}$ & 5 & 43 & 39 & $85(*)$ \\
\hline
\end{tabular}

(*) Indica a relação entre as 33 empresas visitadas em Rio Branco e as 39 serrarias cadastradas, no IBAMA, e atuando nesta cidade

(*) Indicates the ratio among the group of 33 firms visited in Rio Branco and 39 sawmills enrolled at IBAMA and acting in this city

(**) Mostra a relação entre as 43 firmas visitadas e um contigente de 90 unidades atuando em Rio Branco em 1995, segundo informações do setor

(**) Shows the ratio between the 43 visited firms and a group of 90 units acting in Rio Branco in 1995, considering sector information

$\mathrm{Na}$ coleta de dados primários utilizou-se de questionários desenvolvidos segundo sugestões de GIL (1991), TAGLIACARNE (1978) e RICH (1970), cujos detalhes são pormenorizados a seguir.

Vale realçar que realizou-se, com uma amostragem piloto, a um teste do questionário aplicado neste estudo. Com isso, como sugere SPROULL (1988), foi possível: (1) gerar dados num nível desejado e (2) obter, dos entrevistados, uma perfeita interpretação dos itens incluídos no questionário.

\section{Administração de marketing na comercialização do produto final}

$\mathrm{Na}$ análise da administração de marketing abordou-se os seguintes pontos, sugeridos por RICH (1970), LINTU (1984), SINCLAIR (1992) e KOTLER (1994): (1) ambiente de marketing; (2) composto de marketing e (3) mercado-alvo.

O ambiente de marketing, como indica KOTLER (1994), foi analisado pelos os fatores deslocadores da demanda: (1) população; (2) renda; (3) bens substitutos; (4) tecnologia; (5) gosto e (5) estado-da-arte das atividades econômicas.

É válido citar que obteve-se tais informações juntos aos seguintes órgãos: Secretaria Estadual de Planejamento do Estado do Acre (SEPLAM-AC) e no Serviço Brasileiro de Apoio a Pequena e Média Empresa (SEBRAE-AC) 
A abordagem sobre o uso do composto de marketing considerou os seguintes pontos: (1) produto; (2) preço; (3) promoção e serviços especiais.

Em termos de produto, identificou-se e analisou-se: (1) possíveis impactos, para o setor, de uma alteração na oferta de matéria-prima madeireira e (2) quais seriam as barreiras à introdução de novas espécies madeireiras neste mercado.

A tendência, do comprador de Rio Branco, em aceitar novas espécies, foi obtida segundo uma lista de itens (preço, cor, densidade, volume disponível, opções de uso, preço e propriedades físicas), sugerida por GRESHAM (1995), que indicam quais qualidades o consumidor médio mundial espera ter nas espécies madeireiras.

O item preço foi abordado segundo: (1) valores praticados, em 1995, para a madeira (em tora e serrada ou na forma de móveis) e (2) as políticas que o setor adota para fixar o preço de venda dos seus produtos.

Analisou-se, ainda, as políticas, adotadas pelo setor madeireiro local, quanto ao fornecimento de serviços especiais e de divulgação das suas firmas.

A identificação dos mercados alvo foi desenvolvida obtendo-se dos empresários madeireiros locais o destino da comercialização dos seus produtos. Questionou-se ainda estes empresários quanto à atuação ou não de intermediários nos processos de comercialização dos seus produtos, como também se haveriam dificuldades para uma nova empresa entrar no mercado de Rio Branco.

\section{Formulação de uma estratégia de marketing}

A formulação da estratégia para um programa de marketing, por sugestões de RICH (1970), SINCLAIR (1992) e KOTLER (1994), identificou os pontos fracos e fortes, oportunidades e ameaças relacionados ao setor madeireiro de Rio Branco.

Estes pontos estão indicados graficamente na Figura 1. 
Figura 1 - Estrutura da formulação da estratégia competitiva Figure 1- Structure of competitive strategy formulation

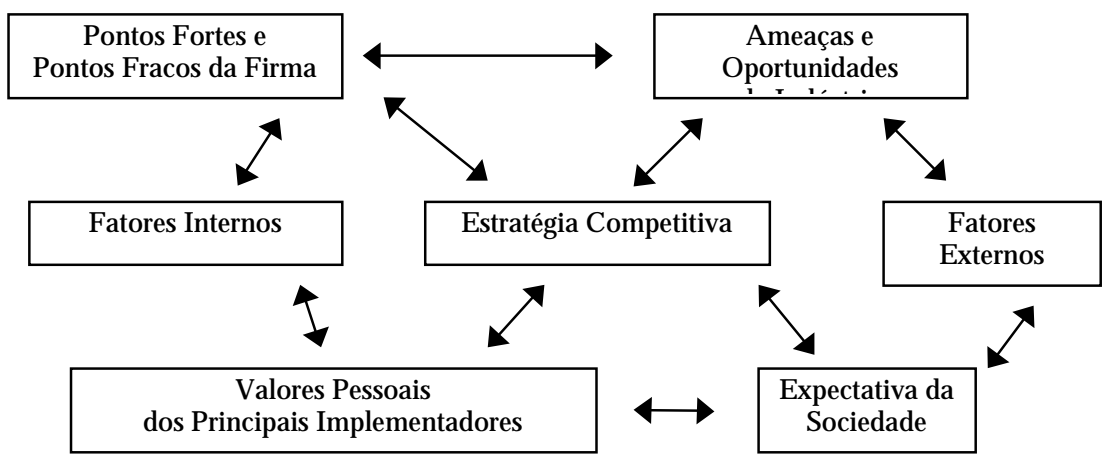

Fonte/Source: PORTER (1986)

\section{RESULTADOS E DISCUSSÃO}

\section{AMBIENTE DE MARKETING}

Uma análise do mercado madeireiro, em Rio Branco, em 1995, permite inferir que: (1) no curto prazo, a população local e sua respectiva renda não devem aumentar significativamente; (2) ações ambientalistas tendem diminuir a oferta de matéria-prima e (3) a industrialização não deve ter um aperfeiçoamento.

Com isso, a opção para o aumento da demanda por madeira deverá ser um programa de marketing junto às empresas de construção civil.

\section{CARACTERIZAÇÃO DA MATÉRIA-PRIMA FLORESTAL Consumo de matéria-prima}

Em 1995, as serrarias e marcenarias de Rio Branco consumiam, em média por unidade fabril, 3.400 e $175 \mathrm{~m}^{3} /$ ano de madeira em tora, respectivamente. Os depósitos, por sua vez, comercializavam $330 \mathrm{~m}^{3}$ de madeira serrada por unidade de revenda (para um total de $4.800 \mathrm{~m}^{3} /$ ano neste segmento).

A Tabela 2 mostra, conforme opiniões dos empresários madeireiros de Rio Branco, o impacto para o setor caso ocorresse uma oferta maior de madeira.

Tabela 2 - Impactos, no setor madeireiro de Rio Branco, caso de um aumento na oferta de matéria-prima madeireira, 1995 
Table 2 -Impacts on the Rio Branco wood sector, in case of an increase in the rawmaterial supply, 1995

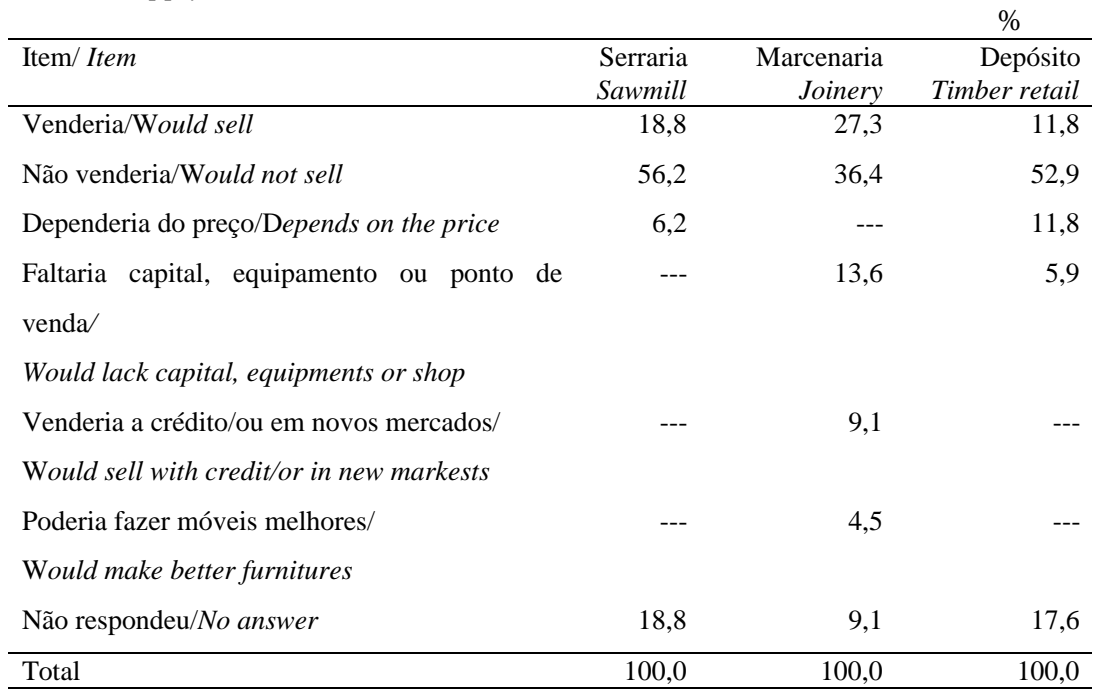

Observa-se na Tabela 2 que aproximadamente a metade dos proprietários de serrarias e depósitos de madeira, não haveria, em Rio Branco, um mercado para uma oferta maior de madeira. No segmento das marcenarias, por outro lado, tem-se que a existência ou não de um mercado local para uma oferta maior de madeira divide a opinião da maioria dos proprietários destas indústrias.

\section{Espécies florestais utilizadas}

Constatou-se que, no período em análise, aproximadamente $89 \%$ das serrarias consumia madeira de cumaru ferro (Dipterex alata Vog), 67\% utilizava cerejeira (Torressea acreana Duke) e 58\% desdobrava cedro (Cedrela $\mathrm{sp})$. Entre as marcenarias, neste período, cedro e cerejeira eram usadas em 83,7 e $97,7 \%$ das indústrias, respectivamente.

A Tabela 3 apresenta pontos básicos, realçados pelo setor madeireiro de Rio Branco, para o sucesso na introdução de novas espécies madeireiras no mercado.

Pelo exposto na Tabela 3, a necessidade de divulgação, para os consumidores, das qualidades das espécies, segundo aproximadamente a metade dos proprietários de serrarias e depósitos de madeira, é um fator importante para o sucesso na introdução de novas espécies madeireiras em Rio Branco.

Tabela 3 - Fatores relevantes na oferta de novas espécies no setor madeireiro de Rio Branco, 1995 
Table 3 - Relevant factors in the supply of new species in the wood sector of Rio Branco, 1995

\begin{tabular}{|c|c|c|c|}
\hline & & & $\%(*)$ \\
\hline Fator/ Factor & Serraria & Marcenaria & Depósito \\
\hline & Sawmill & Joinery & Timber \\
\hline & & & retail \\
\hline Dependeria do preço/Depends on the price & 5,9 & 9,1 & 6,3 \\
\hline Necessitaria de pesquisa/Needs of research & 17,7 & 50,0 & 12,3 \\
\hline Custo alto de exploração/High price of harvesting & 11,8 & --- & 12,5 \\
\hline Necessitaria de divulgação/Needs of divulgation & 47,1 & 40,9 & 43,8 \\
\hline Sem dificuldade/No difficulties & 11,8 & --- & 12,5 \\
\hline Outros/Others & --- & 4,6 & 6,3 \\
\hline Não respondeu/No answer & 23,5 & 18,2 & 25,0 \\
\hline
\end{tabular}

(*) Percentual relacionado ao número de empresas que consideram o referente item como dificuldade ao bom trabalho de uma empresa no setor madeireiro de Rio Branco

(*) Perceptual related to the number of firms which recognize this item as a difficulty for the good performance of the enterprises in the wooden sector of Rio Branco

No segmento de marcenarias, por sua vez, conforme respostas de quase a metade dos seus empresários, a realização de pesquisas tecnológicas quanto as qualidades das novas espécies madeireiras e um posterior esforço de divulgação dos resultados destes estudos, aos potenciais consumidores, são pontos importantes no processo de introdução de novas espécies neste mercado.

A Tabela 4 compara o comportamento do consumidor local, segundo informações dos marceneiros de Rio Branco, com o consumidor mundial médio, conforme GRESHAN (1995), no processo de classificar os fatores que o influenciam na escolha de espécies madeireiras utilizadas em seus móveis.

Observa-se na Tabela 4 que, para o consumidor de Rio Branco, o fator preço da espécie madeireira pesa significativamente na escolha da matériaprima para seus móveis. O consumidor mundial médio, por outro lado, considera mais a cor na escolha da espécie para elaborar seus móveis (o fator preço da madeira, neste grupo de consumidores, ocupa a quinta posição, entre os seis fatores analisados).

Mesmo sendo difícil introduzir novas espécies, não se pode desconsiderar as madeiras "emergentes de mercado". PASTORE JÚNIOR (1995), por exemplo, cita que o tauari (Couratari spp), que era a 23ํㅡㄹ espécie vendida e o curupixá (Micropholis venulosa) que não era exportado pelo Pará em 1987, passaram a ser a primeira e segunda espécie, respectivamente, vendida em 1994 por aquele Estado.

Tabela 4 - Classificação dos fatores na escolha de espécies madeireiras, no setor moveleiro de Rio Branco, 1995 
Table 4 - Classification of factors in the choice of wood species, in the Rio Branco furniture sector, 1995

\begin{tabular}{|c|c|c|c|}
\hline \multirow[t]{2}{*}{ Fator/ Factor } & & \multicolumn{2}{|c|}{$\begin{array}{l}\text { Ordem de prioridade } \\
\text { Priority order }\end{array}$} \\
\hline & & $\begin{array}{l}\text { Consumidor de Rio Branco/ } \\
\text { Rio Branco consumer }\end{array}$ & $\begin{array}{l}\text { Consumidor mundial médio }(*) \\
\text { Average worldwide consumer }\end{array}$ \\
\hline Preço/Price & & 1 & 5 \\
\hline Cor/Colour & & 2 & 1 \\
\hline Peso/Weight & & 3 & 2 \\
\hline $\begin{array}{l}\text { Propriedade física/ } \\
\text { Physical properties }\end{array}$ & & 4 & 6 \\
\hline $\begin{array}{l}\text { Múltiplo uso/ } \\
\text { Multiple use }\end{array}$ & & 5 & 4 \\
\hline $\begin{array}{l}\text { Disponibilidade } \\
\text { mercado/ } \\
\text { Market availability }\end{array}$ & no & 6 & 3 \\
\hline
\end{tabular}

(*) GRESHAN (1995)

\section{Preços praticados para a matéria-prima florestal}

As Tabelas 5 e 6 indicam os preços médios de mercado praticados pelas serrarias e marcenarias respectivamente, para as espécies florestais. 
Tabela 5 - Preço médio da madeira em pé e em tora nas serrarias, Rio Branco, 1995 Table 5 - Average price of stumpage and log in the Rio Branco sawmills, 1995

\begin{tabular}{lcc}
\hline Espécie & $\begin{array}{c}\text { Árvore em pé (US\$/arv.) } \\
\text { Stumpage price (US\$/tree) }\end{array}$ & $\begin{array}{c}\text { Tora posto serraria }\left(\mathrm{US} \$ / \mathrm{m}^{3}\right) \\
\text { Log in the sawmill }\end{array}$ \\
\hline Mogno & --- & 142,99 \\
Cedro & 81,30 & 80,29 \\
Cerejeira & 74,18 & 72,59 \\
Cumaru cetim & 53,42 & 37,27 \\
Cumaru ferro & 45,50 & 37,13 \\
Jatobá & 53,31 & 35,77 \\
Mulateiro & 21,37 & $34,72$. \\
Copaíba & 53,42 & 32,19 \\
Sumaúma & 28,16 & 31,35 \\
Amarelão & 36,89 & 28,89 \\
Sucupira & 31,87 & 26,29 \\
Birindiba & 31,55 & --- \\
Pereira & 42,57 & --- \\
Guaruba & 53,42 & --- \\
Angelim & 53,42 & --- \\
Manitê & --- & 22,00 \\
\hline
\end{tabular}

Vale salientar que, segundo relatos de ARAUJO (1991), em 1990 o preço da árvore em pé, para as espécies de consumo regional e nacional ou internacional era de US $\$ 6,50 / \mathrm{m}^{3}$ e US\$ $15,00 / \mathrm{m}^{3}$, respectivamente. Para a madeira posto serraria, os preços para espécies com mercado nacional e internacional eram de US $\$ 18,00 / \mathrm{m}^{3}$ e US $\$ 60,00 / \mathrm{m}^{3}$ respectivamente. 
Tabela 6 - Preço médio da matéria-prima florestal nas marcenarias de Rio Branco, 1995 Table 6 - Average price of forest raw-material in the Rio Branco joineries, 1995

\begin{tabular}{|c|c|c|c|c|c|c|c|}
\hline \multirow{2}{*}{ Espécie } & \multicolumn{2}{|c|}{ Arvore em pé } & \multirow{2}{*}{$\begin{array}{c}\begin{array}{c}\text { Prancha na } \\
\text { mata }\end{array} \\
\mathrm{US} \$ / \mathrm{m}^{3}\end{array}$} & \multicolumn{2}{|c|}{$\begin{array}{l}\text { Posto movel } \\
\left(\mathrm{US} \$ / \mathrm{m}^{3}\right)\end{array}$} & \multicolumn{2}{|c|}{ Serraria.(US $\left.\$ / \mathrm{m}^{3}\right)$} \\
\hline & $\mathrm{US} \$ / \mathrm{m}^{3}$ & US\$/árv & & Prancha & Em tora & Tora & Tábua \\
\hline \multirow{2}{*}{ Species } & \multicolumn{2}{|c|}{ Stumpage price } & $\begin{array}{l}\text { Block in the } \\
\text { forest }\end{array}$ & \multicolumn{2}{|c|}{$\begin{array}{c}\text { In the furniture } \\
\text { industry }\end{array}$} & \multicolumn{2}{|c|}{ Sawmill } \\
\hline & $U S \$ / m^{3}$ & $\begin{array}{c}\text { US\$/tre } \\
e\end{array}$ & $U S \$ / m^{3}$ & Block & $\log$ & $\log$ & Lumber \\
\hline Mogno & --- & 114,82 & --- & 179,68 & 186,12 & 177,27 & 496,30 \\
\hline Cedro & 34,96 & 57,64 & 58,39 & 107,20 & 88,45 & 148,08 & 168,66 \\
\hline Cerejeira & 25,44 & 57,64 & 58,39 & 105,72 & 88,45 & 150,39 & 185,25 \\
\hline $\begin{array}{l}\text { Cumaru } \\
\text { ferro }\end{array}$ & 11,12 & 31,47 & --- & --- & 40,44 & 115,67 & --- \\
\hline Jatobá & --- & 31,32 & --- & --- & 62,63 & --- & --- \\
\hline Mulateiro & --- & --- & --- & --- & --- & 105,15 & --- \\
\hline Amarelão & --- & --- & --- & 106,27 & --- & --- & --- \\
\hline Sucupira & 11,12 & 31,32 & --- & --- & --- & --- & -- \\
\hline Pereira & --- & 11,12 & --- & --- & --- & --- & --- \\
\hline Angelim & 11,12 & 39,30 & --- & 96,60 & 74,36 & 128,87 & --- \\
\hline Tauari & 3,18 & --- & --- & --- & 31,85 & --- & --- \\
\hline
\end{tabular}

Com relação a forma de aquisição, pela marcenaria, da matéria-prima na serraria, este fato ocorre com muita frequiência entre as empresas entrevistadas. O procedimento mais usado é quando o dono da marcenaria vai a serraria, escolhe a tora e manda serrar, pagando (só pelo processo de serragem da tora, não incluindo no valor da tora em si) o preço médio de US $\$ 24,36 / \mathrm{m}^{3}$ (em tora) desdobrado.

\section{COMERCIALIZAÇÃO DO PRODUTO FINAL Mercados atingidos}

Os mercados local, nacional e internacional, consumiam, respectivamente $46 \%, 29 \%$ e $25 \%$ da produção das serrarias de Rio Branco em 1995.

Neste mesmo ano, a grande maioria das marcenarias vendia para o mercado de Rio Branco (a maior parte delas atendia a clientes moradores no mesmo bairro em que estão localizadas). Os depósitos, por sua vez, atendiam ao consumidor de pequenos volumes, assim como empresas de construção civil, ambos localizados naquela cidade (era pequena a venda para fora do Estado, neste segmento). 
Observou-se, ainda, que os mercados-alvo servem para diferenciar os produtos madeireiros elaborados em Rio Branco. As serrarias que vendem para o mercado externo usam, basicamente, três espécies: mogno (Swietenia macrophylla King), cedro (Cedrela odorata L.) e cerejeira (Torresea acreana Ducke), enquanto que as indústrias voltadas ao mercado local utilizam um número maior de espécies.

Entre as marcenarias existe uma diferenciação no produto final segundo a classe de renda dos seus clientes. Assim certas indústrias elaboram móveis mais simples para clientes de baixa renda, enquanto outras marcenarias industrializam produtos com melhor acabamento para o consumidor de maior nível de renda.

A Figura 2 apresenta o fluxo de madeira, da floresta no setor madeireiro de Rio Branco em 1995.

Figura 2 - Fluxo madeireiro no setor madeireiro, Rio Branco, 1995

Figure 2 - Wooden flow, in the Rio Branco wood sector, 1995

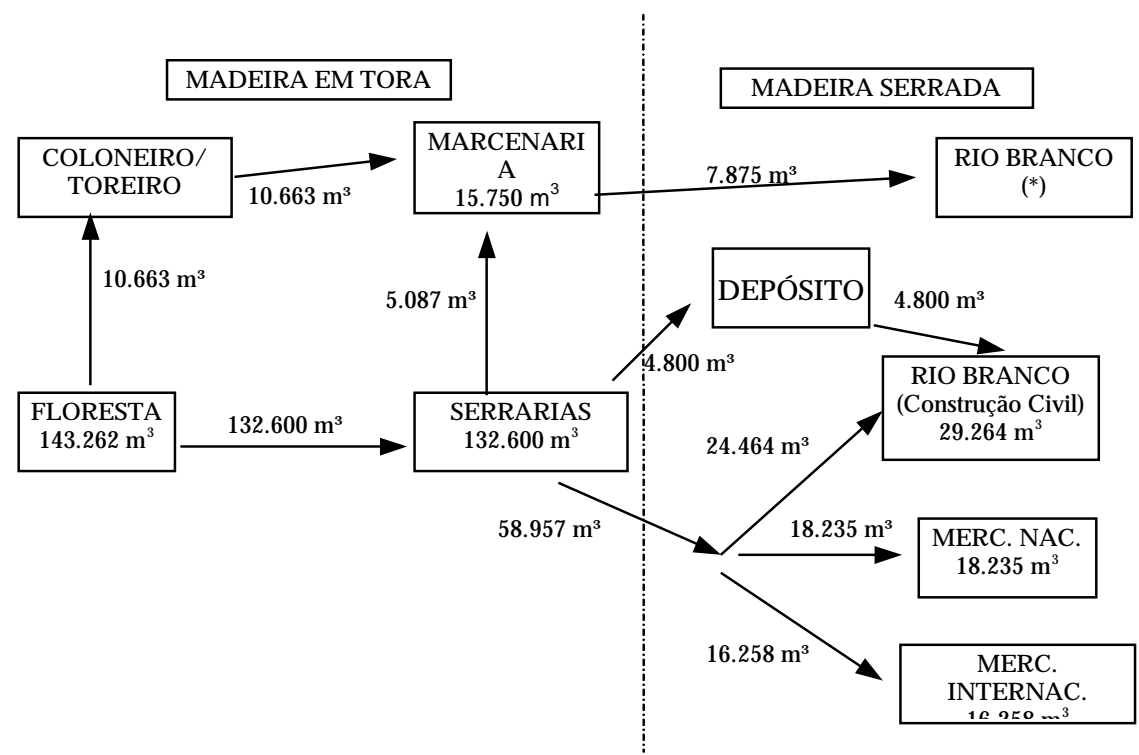

(*) Produção não quantificada por unidade elaborada

(*) Production did not quantify by unit industrialized 


\section{Preço de mercado praticado para o produto final}

As Tabelas 7 e 8 apresentam os preços praticados para os produtos finais das serrarias e marcenarias em Rio Branco, em 1995 respectivamente.

Tabela 7 - Preço médio da madeira serrada, Rio Branco, 1995

Table 7 - Average price of lumber, Rio Branco, 1995

$\mathrm{US} \$ / \mathrm{m}^{3}$

\begin{tabular}{l|r|c|c}
\hline $\begin{array}{l}\text { Espécie } \\
\text { Species }\end{array}$ & $\begin{array}{r}\text { Serraria/ } \\
\text { Sawmill }\end{array}$ & $\begin{array}{c}\text { Depósito/ } \\
\text { Timber retail }\end{array}$ & $\begin{array}{c}\text { Valor médio/ } \\
\text { Average price }\end{array}$ \\
\hline Mogno & 473,98 & 480,26 & 474,60 \\
Cedro & 274,79 & 282,11 & 276,01 \\
Cerejeira & 207,80 & 228,74 & 212,45 \\
Cumaru ferro & 174,70 & 170,58 & 174,33 \\
Sumaúma/ copaíba & 95,04 & $(*)$ & 95,04 \\
Jatobá & 158,39 & 192,10 & 175,25 \\
Cumaru cetim & 143,01 & $(*)$ & 143,01 \\
\hline
\end{tabular}

(*) Valor não obtido

(*) Value not obtained

Comparando os valores indicados na Tabela 7 com os preços amostrados por ARAUJO (1991), tem-se que a madeira para comércio internacional teve um aumento real, entre 1990 e 1995, de $36 \%$ enquanto que o produto para o mercado nacional/local teve uma diminuição real de aproximadamente $12 \%$ em seu preço. 
Tabela 8 - Preço médio dos principais produtos vendidos pelas marcenarias de Rio Branco, 1995

Table 8 - Average price of main products sold by joineries in Rio Branco, 1995

\begin{tabular}{l|c|r}
\hline Produto & $\begin{array}{c}\text { Unidade } \\
\text { Pnit }\end{array}$ & $\begin{array}{c}\text { Valor médio (US\$) } \\
\text { Average price }\end{array}$ \\
\hline Mesa com 4 cadeiras/Table with 4 chairs & $\mathrm{ud}$ & 213,33 \\
Estante/Stand & $\mathrm{ud}$ & 114,98 \\
Guarda roupa/Dresser & $\mathrm{m}^{3}$ & 153,79 \\
Porta/Door & $\mathrm{m}^{2}$ & 37,81 \\
Armário/Cabinet & $\mathrm{m}^{2}$ & 135,02 \\
Cadeira/Chair & $\mathrm{ud}$ & 30,61 \\
Janela/Window & $\mathrm{m}^{2}$ & 38,02 \\
Penteadeira/Dressing table & $\mathrm{ud}$ & 131,00 \\
Berço/Cradle & $\mathrm{ud}$ & 120,70 \\
Componente para cadeira/Chair items & $\mathrm{jog}$ & 15,92 \\
Beliche/Sleeping berth & $\mathrm{ud}$ & 180,07 \\
Cabo de vassoura/ Broomstick & $\mathrm{ud}$ & 0,16 \\
Escrivaninha/Writing desk & $\mathrm{ud}$ & 342,22 \\
Instalação comercial/ & $\mathrm{m}^{2}$ & 227,32 \\
Commercial installation/shop-window & & \\
Moldura/Molding & $\mathrm{m}$ & 6,26 \\
\hline
\end{tabular}

A Tabela 9 apresenta um indicativo de como o setor madeireiro em Rio Branco fixa o preço de venda dos seus produtos. 
Tabela 9 - Critérios adotados na fixação do preço de venda dos produtos no setor madeireiro de Rio Branco, 1995

Table 9 - Criteria adopted in fixation of selling prices of products in the wood sector of Rio Branco, 1995

\begin{tabular}{|c|c|c|c|}
\hline \multirow[b]{2}{*}{ Critério/Criterons } & \multicolumn{3}{|r|}{$(\%)$} \\
\hline & $\begin{array}{l}\text { Serraria } \\
\text { Sawmill }\end{array}$ & $\begin{array}{l}\text { Marcenaria } \\
\text { Joinery }\end{array}$ & $\begin{array}{c}\text { Depósito } \\
\text { Timber retail }\end{array}$ \\
\hline $\begin{array}{l}\text { Adapta-se às condições do mercado/ } \\
\text { Adapt himself to market conditions }\end{array}$ & 17,6 & 4,5 & 6,2 \\
\hline Média/Average & 5,8 & 4,5 & 18,8 \\
\hline $\begin{array}{l}\text { Média mais um desconto/Average plus a } \\
\text { discount }\end{array}$ & 5,9 & 4,5 & -- \\
\hline Média mais custo/Average plus cost & --- & 13,6 & --- \\
\hline Consulta mútua/Consult each other & --- & 4,6 & --- \\
\hline Cliente fornece/Client give information & --- & 4,5 & --- \\
\hline Custo/Cost & 11.8 & 27,3 & 12,5 \\
\hline $\begin{array}{l}\text { Custo mais um percentual de lucro/Cost } \\
\text { plus a percentage of profit }\end{array}$ & --- & 9,1 & 6,2 \\
\hline $\begin{array}{l}\text { Apropr. de custo } \mathrm{p} / \text { compra e média } \mathrm{p} / \\
\text { venda }\end{array}$ & 5,9 & -- & --- \\
\hline $\begin{array}{l}\text { Cost quantification to buying and average } \\
\text { for the selling }\end{array}$ & & & \\
\hline Tabela de preços/Price-list & 41,2 & 4,5 & 31,3 \\
\hline $\begin{array}{l}\text { Vende abaixo da tabela/Sells at price } \\
\text { cheaper than price-list value }\end{array}$ & --- & --- & 12,5 \\
\hline $\begin{array}{l}\text { Varia o preço conforme necessidade de } \\
\text { caixa/Vary the price according to needs of } \\
\text { cash }\end{array}$ & --- & 4,6 & --- \\
\hline Outros/Others & --- & 9,2 & --- \\
\hline Não respondeu/No answer & 11,8 & 9,1 & 12,5 \\
\hline Total & 100,0 & 100,0 & 100,0 \\
\hline
\end{tabular}

Observa-se na Tabela 9, que em 41,2\% das serrarias o preço de mercado da madeira serrada segue uma tabela fixada pelo Sindicato dos Madeireiros de Rio Branco. Salienta-se que $11,8 \%$ das serrarias fixam o preço dos seus produtos segundo seu custo de produção, enquanto que 17,6\% dimensionam seu preço em função das condições do mercado.

No setor moveleiro, $27,3 \%$ das indústrias fixam seus preços em função dos seus custos de produção e 13,6\% determinam preços considerando além dos custos de produção, uma média de preços praticados por seus concorrentes.

Entre os depósitos de Rio Branco, tem-se que 18,8\% destas empresas fixam seus preços segundo a média dos valores praticados por seus concorrentes (em certos casos os empresários chegam a "consultar" preços entre si). Ressalta-se que $31,3 \%$ dos depósitos, a exemplos das serrarias, 
determinam seus preços seguindo a tabela fixada pelo Sindicato Madeireiro de Rio Branco.

Por ultimo, vale ressaltar que os depósitos não verticalizados $(6,2 \%$ da amostra) adotam preços de venda em função do preço de compra dos seus produtos e uma margem de lucro fixada (algumas serrarias pedem ao depósito que revenda seus produtos no mesmo preço posto serraria dando ao depósito entre 10 a $20 \%$ do valor da venda, a título de comissão).

\section{Formas de comercialização}

A forma como os produtos eram comercializados nas serrarias, marcenarias e depósitos em Rio Branco, em 1995, era tanto à vista como a prazo.

Na maioria das vezes, o comprador local adquiria produtos sem a interferência de intermediário. A venda para fora do Estado, por outro lado, podia ser direta (via fax ou telefone com a formulação do pedido de compra ou por parte do vendedor ofertando o produto ou do comprador solicitando a remessa) ou com a vinda de um comprador/representante da matriz da empresa "importadora".

\section{Política de fornecimento de serviços especiais}

A Tabela 10 apresenta os serviços especiais adotados nas serrarias, marcenarias e depósitos de Rio Branco.

Nota-se que, em Rio Branco, 52,9\% das serrarias concedia um desconto no preço de venda, $64,7 \%$ levava o produto no local indicado pelo cliente e $17,6 \%$ considerava que praticar promoções poderia dar prejuízo.

Salienta-se que $17,7 \%$ das serrarias, $27,3 \%$ das marcenarias e $37,5 \%$ dos depósitos divulgam seus produtos no rádio, televisão (tais procedimentos podem, segundo os entrevistados, aumentar as vendas em até $10 \%$, em relação ao comércio sem nenhuma destas estratégias). 
Tabela 10 - Serviços especiais praticados no setor madeireiro, Rio Branco, 1995

Table 10 - Special services in the wood sector, Rio Branco, 1995

\begin{tabular}{|c|c|c|c|}
\hline Procedimento/Procedure & $\begin{array}{l}\text { Serraria/ } \\
\text { Sawmill }\end{array}$ & $\begin{array}{l}\text { Marcenaria/ } \\
\text { Joinery }\end{array}$ & $\begin{array}{l}\text { Depósito/ } \\
\text { Timber } \\
\text { retail }\end{array}$ \\
\hline $\begin{array}{l}\text { Não realiza nenhum serviço especial/ } \\
\text { Do not realize any special service }\end{array}$ & 17.6 & --- & 12,5 \\
\hline $\begin{array}{l}\text { Dá desconto nas vendas à vista/ } \\
\text { Give cash discount }\end{array}$ & 52,9 & 68,2 & 50,0 \\
\hline Fornece frete/Has delivery service & 64,7 & 77,2 & 62,5 \\
\hline Entrega imediata/Immediate delivery & --- & --- & 6,3 \\
\hline $\begin{array}{l}\text { Propaganda em lista telefônica, rádio ou TV/ } \\
\text { Propaganda in telephone list, radio or tv }\end{array}$ & 17,7 & 27,3 & 37,5 \\
\hline $\begin{array}{l}\text { Dá desconto quando não fornece frete/ } \\
\text { Give discount when does not supply } \\
\text { freightage }\end{array}$ & 5,9 & --- & 6,3 \\
\hline Vende à crédito/Sell on credit & 5,9 & 9,1 & 12,5 \\
\hline $\begin{array}{l}\text { Realiza promoções diversas/ } \\
\text { Realize several promotion }\end{array}$ & 11,8 & --- & 6,3 \\
\hline $\begin{array}{l}\text { Garante a qualidade ou concerta o } \\
\text { produto/Warrant quality or repair the product }\end{array}$ & --- & 18,2 & --- \\
\hline $\begin{array}{l}\text { Distribui cartão de visita/ } \\
\text { Distribute visiting-card }\end{array}$ & --- & 9,1 & --- \\
\hline Distribui calendário/Distribute calendar & --- & 4,6 & --- \\
\hline $\begin{array}{l}\text { Faz visita a possíveis clientes/ } \\
\text { Visit possible clients }\end{array}$ & --- & --- & 6,3 \\
\hline Não respondeu/No ansewer & 11,8 & 22,7 & 12,5 \\
\hline
\end{tabular}

(*) Percentual referente ao número de empresas que consideram o mencionado item como dificuldade ao bom trabalho de uma empresa no setor madeireiro de Rio Branco

(*) Percentage related to the number of firms which recognizes this item as difficulty to the good performance of the enterprises in the wooden sector of Rio Branco

Observa-se ainda que $77,2 \%$ das marcenarias entrega montado o produto no local solicitado pelo freguês, a depender do valor da venda, $68,2 \%$ dá desconto (10-20\% em relação ao preço a prazo) e 18,2\% destas empresas garante a qualidade do móvel (até o período de um ano após a compra), ou o repõe no caso de defeitos.

Entre os depósitos de Rio Branco, 50\% destas empresas dá desconto (5-10\%, na venda à vista) e $62,5 \%$ leva o produto no local solicitado (se possível, a entrega é imediata). 
A Tabela 11 apresenta, segundo informações dos membros do setor madeireiro de Rio Branco, os obstáculos à entrada de novas empresas no mercado.

Tabela 11 - Obstáculos a entradas de novas empresas no setor madeireiro

Table 11 - Barriers to entry of new enterprises in the wood sector

\begin{tabular}{|c|c|c|c|}
\hline Item/ Item & $\begin{array}{l}\text { Serraria/ } \\
\text { Sawmill }\end{array}$ & $\begin{array}{l}\text { Marcenaria/ } \\
\text { Joinery }\end{array}$ & $\begin{array}{c}\text { Depósito/ } \\
\text { Timber } \\
\text { retail }\end{array}$ \\
\hline Sem dificuldade/No difficulty & 29,4 & 4,5 & 18,8 \\
\hline Talvez haja dificuldade/ & 5,9 & 4,5 & 6,3 \\
\hline Perhaps occur difficulties & & & \\
\hline Falta de mercado/lack of market & 35,3 & 36,4 & 31,3 \\
\hline $\begin{array}{l}\text { Dificuldade com a oferta/Preço da } \\
\text { madeira/ } \\
\text { Difficulty with wood supply/price }\end{array}$ & 5,9 & 22,6 & 6,3 \\
\hline $\begin{array}{l}\text { Falta de mão-de-obra qualificada/ } \\
\text { Lack of skilled man-power }\end{array}$ & --- & 9,1 & --- \\
\hline $\begin{array}{l}\text { Necessidade de divulgação/ } \\
\text { Necessity of divulation }\end{array}$ & --- & 18,1 & 6,3 \\
\hline Falta de energia/Shortage of energy & --- & 4,5 & --- \\
\hline Imposto/Tax & --- & 4,5 & --- \\
\hline Necessidade de capital/Necessity of capital & 23,5 & 9,1 & 6,3 \\
\hline Concorrência ilegal/Unlawful competition & 11,8 & 4,1 & 12,5 \\
\hline $\begin{array}{l}\text { Concorrência c/ Rondônia e Mato Grosso/ } \\
\text { Competition with Rondônia and Mato }\end{array}$ & 35,8 & 4,5 & 41,0 \\
\hline $\begin{array}{l}\text { Grosso States } \\
\text { Baixa qualidade dos móveis/ } \\
\text { Low quality of furnitures }\end{array}$ & 11,8 & 9,1 & --- \\
\hline $\begin{array}{l}\text { Burocracia excessiva/ } \\
\text { Excess of bureaucracy }\end{array}$ & 5,9 & 4,5 & --- \\
\hline $\begin{array}{l}\text { Falta de incentivo do Governo/ } \\
\text { Lack of Govern incentives }\end{array}$ & 5,9 & 4,5 & --- \\
\hline $\begin{array}{l}\text { Necessidade de verticalização/ } \\
\text { Necessity of verticalization }\end{array}$ & 5,9 & 4,5 & 12,5 \\
\hline Outros/Others & --- & 4,5 & 6,5 \\
\hline Não respondeu/No answer & 11,7 & 9,1 & 18,8 \\
\hline
\end{tabular}

(*) Percentual referente ao número de empresas que consideram o mencionado item como dificuldade ao bom trabalho de uma empresa no setor madeireiro de Rio Branco

(*) Percentage related to the number of firms which recognizes this item as difficulty to the good performance of the enterprises in the wooden sector of Rio Branco

Observa-se na Tabela 11 que, nos segmentos de serrarias e depósitos de madeira, um mercado pequeno dificulta a venda local de madeira serrada. A concorrência com regiões de menor custo de frete, melhor produto e um menor preço de pauta, por sua vez, dificultam vender o produto acreano para o sul do 
país (justificando o pequeno volume da venda de madeira serrada para fora do Estado). Acrescenta-se que as serrarias necessitam de capital para estocar a madeira explorada durante o verão.

No segmento de marcenaria constata-se que pode faltar madeira, peças de reposição, energia e mão-de-obra qualificada às novas indústrias em Rio Branco. Além disso, deve-se vender a prazo (a maioria das marcenarias vende para clientes de baixa renda).

Salienta-se que entre as marcenarias, mesmo com o Serviço SEBRAE/FIEAC organizando feiras anuais de móveis, observou-se que um grupo destas indústrias sente a falta de um local para expor seus produtos.

\section{O CONSUMIDOR DE RIO BRANCO E O PRODUTO CERTIFICADO.}

Pelos depoimentos e pontos de vista obtidos, tem-se que a "certificação de origem" seja, ainda, um termo de pouco conhecimento do madeireiro de Rio Branco.

\section{Existência de preferência por produtos com certificação de origem}

A Tabela 12 indica, segundo membros do setor madeireiro de Rio Branco, o comportamento do consumidor local quanto à preferência de uso entre um produto oriundo de área manejada e um outro proveniente de uma área de desmate. 
Tabela 12 - Preferência do consumidor por um produto com certificado de origem sustentável, Rio Branco, 1995

Table 12 - Consumer preference for products with certification

\begin{tabular}{|c|c|c|c|}
\hline Preferência/Preference & $\begin{array}{l}\text { Serraria/ } \\
\text { Sawmill }\end{array}$ & $\begin{array}{l}\text { Marcenaria/ } \\
\text { Joinery }\end{array}$ & $\begin{array}{l}\text { Depósito/ } \\
\text { Timber } \\
\text { retail }\end{array}$ \\
\hline $\begin{array}{l}\text { O consumidor não interessa-se pela origem } \\
\text { da madeira/ } \\
\text { The consumer has not interest by wooden } \\
\text { origem }\end{array}$ & 58,8 & 27,3 & 52,9 \\
\hline $\begin{array}{l}\text { Consumidor aceitaria (estaria seguro } \\
\text { quanto a origem da madeira)/The } \\
\text { consumer would accept (he would be } \\
\text { secure in concerning the wooden origem) }\end{array}$ & 5,9 & --- & 5,9 \\
\hline $\begin{array}{l}\text { A venda dependeria do preço da madeira/ } \\
\text { The selling would depends on of wooden } \\
\text { price }\end{array}$ & 5,9 & 4,6 & 5,9 \\
\hline $\begin{array}{l}\text { O consumidor interessa-se só pela } \\
\text { qualidade da madeira/The consumer has } \\
\text { only interest by the wooden quality }\end{array}$ & --- & 9,1 & --- \\
\hline $\begin{array}{l}\text { Para o cliente independe a origem } / \text { For the } \\
\text { consumer does not depend the wooden } \\
\text { origem }\end{array}$ & --- & 9,1 & --- \\
\hline $\begin{array}{l}\text { O consumidor, certamente, preferiria o } \\
\text { produto certificado/The consumer, } \\
\text { certally, would prefer a certified timber }\end{array}$ & --- & 4,5 & 5,9 \\
\hline $\begin{array}{l}\text { Talvez só } 1 \% \text { dos compradores preferiria o } \\
\text { produto certificado/ }\end{array}$ & --- & 4,5 & --- \\
\hline $\begin{array}{l}\text { Perhaps } 1 \% \text { out of consumers would } \\
\text { prefer, a certified product }\end{array}$ & & & \\
\hline $\begin{array}{l}\text { Talvez o consumidor preferiria o produto } \\
\text { certificado/ } \\
\text { Perhaps the consumer could prefer a } \\
\text { certified product }\end{array}$ & --- & 4,5 & 5,9 \\
\hline Não respondeu/No answer & 29,4 & 36,4 & 23,5 \\
\hline Total & 100,0 & 100,0 & 100,0 \\
\hline
\end{tabular}

Observa-se nesta tabela que o consumidor de madeira ou móvel em Rio Branco não preocupa-se com o aspecto da origem do produto (este consumidor importa-se apenas com a qualidade da madeira que adquire). 
A Tabela 13 apresenta um indicativo, segundo integrantes do setor madeireiro de Rio Branco, do comportamento do seu consumidor quanto a um aumento no preço deste produto devido ao fato de ter área manejada na origem.

Tabela 13 - Comportamento do consumidor a um preço maior da madeira vinda de uma área manejada, Rio Branco, 1995

Table 13 - Consumer's behavior to a higher price of the wood coming from a certified area

\begin{tabular}{lrrr}
\hline Comportamento/Behavior & $\begin{array}{r}\text { Serraria/ } \\
\text { sawmill }\end{array}$ & $\begin{array}{c}\text { Marcenaria/ } \\
\text { Joinery }\end{array}$ & $\begin{array}{c}\text { Depósito/ } \\
\text { timber retail }\end{array}$ \\
\hline Não pagaria/Would not pay & 70,6 & 45,5 & 58,8 \\
Talvez o consumidor informado pagaria/ & 5,9 & 13,6 & 12,5 \\
Perhaps the awarded consumer would pay & & & \\
Não respondeu/No answer & 23,5 & 40,9 & 18,7 \\
\hline Total & 100,0 & 100,0 & 100,0 \\
\hline
\end{tabular}

Observa-se, nesta Tabela, que a grande maioria do consumidor de Rio Branco não pagaria a mais por um produto com origem de certificação.

\section{Impacto do preço da madeira certificada no seu consumo em Rio Branco}

De um modo geral, os empresários do setor madeireiro de Rio Branco não sabem quantificar o impacto no consumo de madeira devido a adoção do manejo florestal (uma operação mais cara que a atual forma de extração madeireira).

Há, entretanto quem afirme que, caso todas as empresas aumentem seus preços, não haverá uma mudança no consumo. Outros projetam uma queda de $1 \%$ na venda para um aumento de $1 \%$ no preço da madeira (tem-se que este setor, quando de reajustes de preço, leva de 15 a 20 dias para nivelar a venda).

É importante salientar que MOHAMED, MOHD \& GHANI (1995) comentam que, na escolha por produtos madeireiros de origem tropical, cada vez mais os consumidores buscam produto vindo de área manejada.

Acrescenta-se que BOURKE \& BOURKE (1996) relacionam a certificação de origem da madeira ao marketing (e não ao manejo florestal) e consideram que, internacionalmente, a certificação influenciará em muito o marketing.

\section{PONTOS ESTRATÉGICOS DO SETOR MADEIREIRO DE RIO BRANCO}

Pelo ambiente de marketing apresentado, pode-se afirmar que ao se propor uma estratégia de marketing para o Setor Madeireiro de Rio Branco deve-se considerar: 


\section{Pontos fracos}

Um sistema de manejo para a produção florestal sustentada beneficiará a venda, no curto prazo, apenas para mercados externos a Rio Branco. No mercado local, há a concorrência com madeira mais barata vinda de áreas de desmate.

Há uma escassez em mão-de-obra qualificada que atenda aos processos produtivos, principalmente para industrializar móveis de alta qualidade.

A distância com os grandes centros, Rio ou São Paulo e Paraná, encarece o custo de frete, devendo-se, assim transportar via Manaus e/ou Belém.

\section{Pontos fortes}

A divulgação, para consumidores externos, que no Acre se executa o manejo florestal e o possível comércio, via Pacífico (pelo Acre ou Rondônia), podem tornar-se pontos fortes no planejamento para a venda da madeira vinda deste Estado.

\section{Ameaças}

Considerando que, entre as serrarias e consumidores externos ao Acre, já está mais difundido o mecanismo de certificação de origem da madeira, poderá colocar aquelas indústrias em vantagem competitiva em relação às firmas acreanas na busca por mercado.

\section{Oportunidades}

O fato da ITTO financiar projetos na região pode ser capitalizado no sentido de que esta organização divulgue o trabalho feito neste Estado (sendo, de certa forma como um "certificado informal de origem do produto).

O potencial florestal madeireiro necessita apenas de uma divulgação criteriosa das qualidades das madeiras da região.

\section{CONCLUSÕES}

Em função do cenário, descrito anteriormente, pode-se inferir os seguintes pontos em relação ao setor madeireiro de Rio Branco:

- No sentido de assegurar uma demanda para o produto madeireiro acreano, esforços de marketing devem ser desenvolvidos pelos empresários locais juntos as firmas de construção civil de Rio Branco;

- O atual estágio em que se encontram as indústrias deste setor demanda certas medidas administrativas (aprendizado e adoção de técnicas de marketing), técnicas-operacionais (treinamento de mão-de-obra, atualização, melhoria da infra-estrutura industrial e implementação de um sistema de controle e 
apropriação de custos) e políticas (um melhor relacionamento com o setor público, enfatizando a importância do setor para a região, justificando um melhor atendimento de suas necessidades);

- A baixa estrutura organizacional das indústrias do setor não permite a pratica das técnicas de marketing (uso adequado dos compostos de marketing) para um sucesso econômico aceitável deste setor;

- A inexistência da prática do manejo florestal generalizada, entre as firmas madeireiras locais, pode se caracterizar como um obstáculo às serrarias de Rio Branco quando da comercialização com mercados externos, onde itens como certificado de origem são pontos chaves para a negociação de produtos madeireiros.

\section{BIBLIOGRAFIA CITADA}

AMARO, M.A.; SILVA, Z.A.G.P. da G. \& BRAZ, E.M. . Diagnóstico do setor florestal do Estado do Acre. Rio Branco, UFAC. In: Encontro de Pesquisadores da Amazônia, 7. Anais.... 1993. p.135.

ARAUJO, H.J.B. Diagnóstico das indústrias de serrarias do Estado do Acre. Rio Branco: FUNTAC, 1991. 238 p. (não publicado).

BOURKE, J. \& BORKE I.J. Global trends in marketing of environmentally certified forest products. Camberra, Australian Bureau of Agricultural and Resource Economics (ABARE) In: Outlook 96 commodity markets \& resource management. Volume 1, Proceedings (National Agricultural and Resources Outlook Conference). 1996. p. 168-176.

COHEN, D.H. \& SMITH, P.M. Global marketing strategies for forest product industries. Canadian-Journal-of-Forest-Research. 22: 1, p. 124-131.

DEPARTAMENTO DE PESQUISA TECNOLÓGICA DO RECURSOS NATURAIS-DPTRN. Diagnóstico das indústrias de serrarias da Região. de Rio Branco, Rio Branco: FUNTAC, 1986. 238 p.

EASTIN, I.L. Marketing strategies to promote sustainable forest management in tropical África. FPRDI Jounal. 22:1, p. 79-88.

FEDERAÇÃO DAS INDÚSTRIAS DO ESTADO DO ACRE-FEIAC \& FUNDAÇÃO DE TECNOLOGIA DO ESTADO DO ACRE-FUNTAC. Diagnóstico das indústrias moveleiras de Rio Branco. Rio Branco: FIEAC/FUNTAC. $1992.110 \mathrm{p}$.

FUNDAÇÃO DE TECNOLOGIA DO ESTADO DO ACRE-FUNTAC. Diagnóstico das indústrias de serraria de Rio Branco. Rio Branco: FUNTAC, 1990. 157p.

. Levantamento do desperdício de madeira existente nas marcenarias de Rio Branco: possibilidade de utilização do resíduo de madeira para 
fabricação de novos produtos. Rio Branco: SEBRAE/FUNTAC, 1994. 33 p.

GHAZALI, B. H. \& SIMULA, M. Certification: Who will pay the price?

Forest Update, V.4, N.5. p. 3-5.

GIL, A.C. Técnicas de pesquisa em economia. São Paulo: Atlas, 1991. 195 p.

GRESHAM, G. In quest of the unknown. ITTO Tropical Forest Update. V. 5 N. 2, p. 3-5.

HUMMEL, A. C. et al. Diagnóstico do subsetor madeireiro do Estado do Amazonas. Manaus: SEBRAE/AM-IMA/AM, 1994. 76 p.

INSTITUTO BRASILEIRO DE DESENVOLVIMENTO FLORESTAL-IBDF.

Análise do setor industrial florestal: Acre-Rondônia. Rio de Janeiro: IBDF, 1984. $92 \mathrm{p}$.

Identificação e agrupamento de espécies de madeiras tropicais Amazônica. Brasília: IBDF. 1985. 59 p.

LAFLEUR, J.; SINGHI, P.M. \& MAcDIKEN, K.G. Marketing in forestry and agroforestry by rural people. Banglok : FAO Regional Office for Asia and the Pacific. 1996. 56 p.

LINTU, L. Marketing in the forestry sector. Unasylva, 38: 3, p. 10-19. 1986a

Marketing and marketing management. In: FOOD AND AGRICULTURE ORGANIZATION OF THE UNITED NATIONS. Management of forest industries. Rome: FAO, 1986b. p. 223-240.

MOHAMED, S.; RUSLI, M. \& GHANI, A. Wood products from sustainably managed forests: consumer preferences. Options-Serdang. 10: 1, p. 13-14.

NIEMELA, J.S. \& SMITH, P.M. A cross-national investigation of softwood sawmill marketing strategies. Forest-Science. 42: 3, p. 290-299.

PASTORE JÚNIOR, F. Las especies menos utilizadas: Un experimento brasileño. Actualidad Forestal Tropical. V.3. N. 2. p.11

PATNAIK, A. Marketing of farm forestry produce: a new challenge for farmers. Indian-Forester. 122: 7, p. 637-640.

PAUN, D.A.; SHOOK, S.R. \& SCHREUDER, S.R. Educational mindsets: the importance of marketing and economics in forestry education. Journal of Forestry. 94:9, p. 29-33.

PORTE, M.E. Estratégia competitiva: Técnica para análise de indústrias e da concorrência. Rio de Janeiro: Editora Campus Ltda, 1986. 362 p.

PRICE, C. Theory and aplication of forest economics. Bangor: Basil Blackwell Ltd, 1989. 402 p.

RICH, S.U. Marketing of forest products: Text and cases. New York: McGraw-Hill Book Company. 1970. 712 p.

SAMANEZ MERCADO, R. Timber production and marketing in the Brazilian Amazon. East Lansing, 1980. Tese (Doutor em Filosofia, Michigan State University, 1980. 131 p. 
SANTOS, J. Situação da indústria madeireira no Município de Manaus (1981 e 1983) e das serrarias do Estado do Amazonas (1981) .Curitiba, UFPR, Dissertação de Mestrado, 1986, 78 p.

SERVIÇO BRASILEIRO DE APOIO ÀS MÉDIAS E PEQUENAS EMPRESAS - SEBRAE. Como montar marcenaria. Brasília: SEBRAE, 1993. $25 \mathrm{p}$.

SILVA, J.C.G. E. da Análise da formulação de estratégias de marketing internacional de empresas de papel e celulose. Piracicaba, 1996. Tese (Doutorado em Ciência Aplicada) - Escola Superior de Agricultura Luis de Queiroz, Universidade de São Paulo.

SILVA, Z.A.G.P. da G.. Subsídios técnicos para a elaboração de uma estratégia de marketing para bens madeireiros produzidos no Estado do Acre. Rio Branco: FUNTAC, 1996.

. \& TOMASELLI, I. Economics analysis on the wooden sector in the State of Acre-Brazil, 1995. In: International Symposium Forest Resource Economics. Umeä/Suécia, Resumo. Umeä School of Business and Economics/Department of Forest Economics/SLU-Umeä/IUFRO Division VI S6.16-00, 1997.

SPROULL, N. L. Handbook of research methods: A guide for practitioners in the social sciences, New Jersey: The Scarecrow Press, Inc., 1988. $404 \mathrm{p}$ TAGliaCARne, G. Pesquisa de Mercado: Técnica e Prática. São Paulo: Atlas. 1978. 469 p. 